\title{
NEUTRON MODERATION
}

BY ACOUSTIC MODES OF METAL HYDRIDES

By

E. U. VAUGHAN

\section{ATOMICS INTERNATIONAL}
A DIVISION OF NORTH AMERICAN AVIATION, INC.
P.O. BOX 309
CANOGA PARK, CALIFORNIA

CONTRACT: AT(11-1)-GEN-8

ISSUED: OEC 1959 


\section{DISCLAIMER}

This report was prepared as an account of work sponsored by an agency of the United States Government. Neither the United States Government nor any agency Thereof, nor any of their employees, makes any warranty, express or implied, or assumes any legal liability or responsibility for the accuracy, completeness, or usefulness of any information, apparatus, product, or process disclosed, or represents that its use would not infringe privately owned rights. Reference herein to any specific commercial product, process, or service by trade name, trademark, manufacturer, or otherwise does not necessarily constitute or imply its endorsement, recommendation, or favoring by the United States Government or any agency thereof. The views and opinions of authors expressed herein do not necessarily state or reflect those of the United States Government or any agency thereof. 


\section{DISCLAIMER}

Portions of this document may be illegible in electronic image products. Images are produced from the best available original document. 


\section{$A$,}

\section{DISTRIBUTION}

This report has been distributed according to the category "Physics and Mathematics" as given in "Standard Distribution Lists for Unclassified Scientific and Technical Reports" TID-4500 (14th Ed.), October 1, 1958. A total of 650 copies was printed.

\section{ACKNOWLEDGMENT}

The author of this report has had the benefit of comments by C. Warner and E. R. Cohen on the first draft, and especially of conversations with G. W. Lehman which led to the frequency estimate of section II. 


\section{(A)}

\section{CONTENTS}

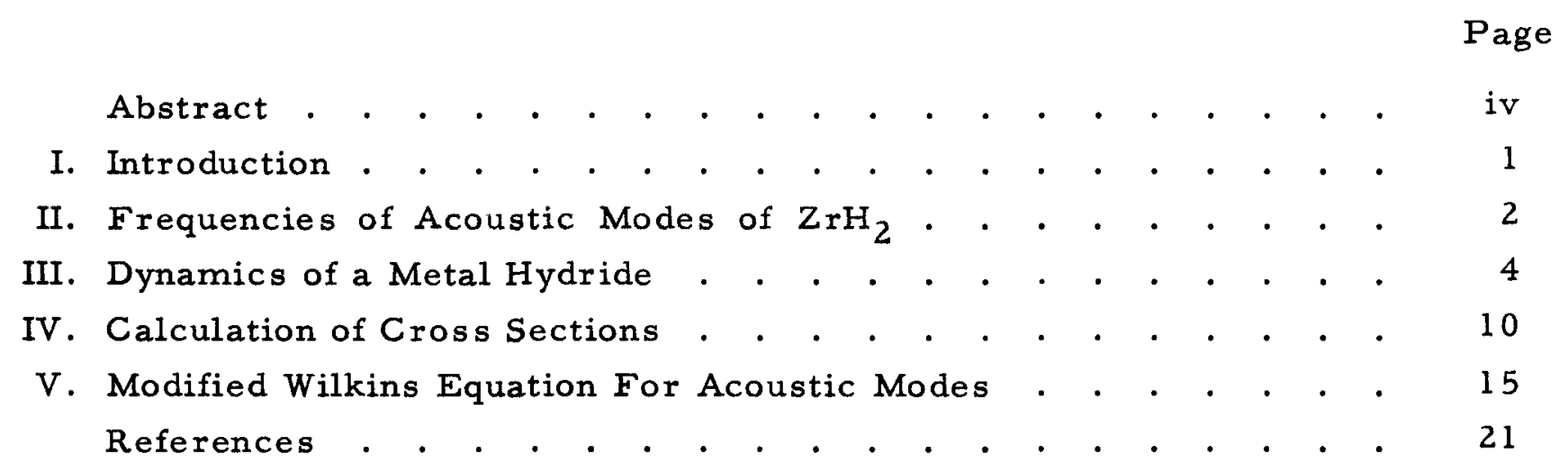




\title{
Al
}

\begin{abstract}
The excitation by nuclear collisions of the acoustic modes of a metal hydride crystal has been investigated, using a model of the crystal based on experiments on $\mathrm{ZrH}_{2}$, but slightly more general. It is found that these modes contribute little to neutron moderation in $\mathrm{ZrH}_{2}$. In the course of the discussion, a generalized form of the Wilkins equation, which determines the spectrum of neutrons thermalizing in a heavy moderator, is developed, applicable when the scattering cross section varies with energy.
\end{abstract}




\section{INTRODUCTION}

Work previously reported ${ }^{1,2}$ on moderation by harmonically bound protons has been based on the optical modes observed in experiments ${ }^{3,4}$ on the inelastic scattering of cold neutrons by $\mathrm{ZrH}_{2}$. The purpose of the work reported herein is to show that the acoustical modes which must also exist do not in general significantly modify the earlier conclusions.

Because of these modes, the energy transfer in a collision need not be exactly an integer multiple of the $h \nu$ of the optic modes. The optic "line" is broadened by the Doppler effect arising from the motion imposed on the struck proton by the acoustic modes. As a result, the sharp corners produced in the neutron spectrum at integer multiples of $h \nu$ by the optic modes alone ${ }^{1,2}$ are rounded off; in fact, they are probably obliterated except the large one at $E=h \nu$. This change in the spectrum is minor, as is expected from the small moderating power of the heavy metal atoms compared to the protons.

The acoustic modes appear to have a more important effect on neutrons of energy less than $h \nu$, which are unable to lose energy to the optic modes, but can lose energy to the acoustic modes. The importance of the acoustic modes for these slow neutrons is to be measured relative to the only remaining competing processes of leakage and absorption. It will appear that these processes, even if small compared with moderation by the optic modes, are large enough to dominate moderation by the acoustic modes. 


\section{FREQUENCIES OF ACOUSTIC MODES OF $\mathrm{ZrH}_{2}$}

It is necessary to begin by determining the range of frequencies spanned by the acoustic modes. The experiments on the optic modes ${ }^{4}$ show a peak due to the acoustic modes. Because of multiple-phonon processes, this peak gives only an upper limit for the higher acoustic frequencies. This upper limit is about $0.020 \mathrm{ev}$. Estimates of acoustic frequencies are often based on measurements of elastic moduli or of low temperature specific heats, but such information is not available for highly-hydrided zirconium hydride. The basis of the following estimate is that frequencies depend on masses and forces, that the masses of hydrogen and zirconium are known, and that much the same forces act on the protons as on the zirconium atoms; consequently, the acoustic frequencies can be estimated in terms of the known optical frequency.

The estimate is limited in accuracy because the nature of the forces and of the modes is not well known. The narrow range of optical frequencies seems to show that proton-proton forces are not important, and it may be assumed that this is true of zirconium-zirconium forces as well. In any case, the protonzirconium forces are expected to dominate because the shortest proton-zirconium distance is less than the shortest proton-proton and zirconium-zirconium distances.

The structure of $\mathrm{ZrH}_{2}$ is, to an accuracy sufficient for the present purpose, a face-centered cubic lattice of zirconium atoms with protons in the tetrahedral interstices. ${ }^{5}$ Thus each proton has four nearest-neighbor zirconium atoms, while each zirconium atom has eight nearest-neighbor protons and twelve nearest-neighbor zirconium atoms.

The optical modes are simple harmonic oscillations of protons relative to their four nearest-neighbor zirconiums; thus, the restoring force is that of four proton-zirconium bonds, and the mass is essentially that of a proton. The acoustic modes are motions of the zirconium atoms relative to each other, the protons with their much smaller inertia merely adjusting themselves to the instantaneous state of the zirconium lattice. The highest-frequency acoustic modes are waves in which neighboring zirconium atoms are nearly $180^{\circ}$ out of phase; hence the effective mass in these modes is roughly the reduced mass 
of two zirconium atoms, or half the mass of one such atom. The bonds affected by the motion of one zirconium atom are those which join it to its eight nearestneighbor protons, together with those joining these protons to its twelve nearestneighbor zirconium atoms. - a total of 32 bonds, or $32 / 12=8 / 3$ bonds per nearest-neighbor zirconiúm. Thus, only about $3 / 8$ of the displacement of a zirconium atom relative to its neighbors can be assigned to any one protonzirconium bond in acoustic modes, whereas the entire displacement can be so assigned in optical modes.

The above rough argument suggests that the restoring forces in the highfrequency acoustic modes are perhaps $3 / 8$ of those in the optic modes, while the masses are increased by a factor of half the zirconium-proton mass ratio. As the frequency of an oscillation varies as the square rooto of the ratio of restoring force to mass, the resulting ratio may be about

$$
\frac{(h \nu)_{a c}}{(h \nu)_{o p}} \approx \sqrt{\frac{3 / 8}{91 / 2}} \approx \frac{1}{11}
$$

(using 91 as the mass of zirconium). The optical frequency is 4

$$
(h \nu)_{o p}=0.13 e v ;
$$

hence the acoustic frequency is

$$
(h \nu)_{a c} \approx 0.012 \xi v
$$

which is about $k T / 2$ at room temperature.

While the above estimates of masses and forces are not unexceptionable, the error in the frequency ratio is probably not very large, especially in view of the square roots involved. The conclusion that the quantum energy of the highest acoustic frequencies is decidedly less than thermal energy, even at temperatures as low as room temperature, seems reasonably secure. 


\section{DYNAMICS OF A METAL HYDRIDE}

The dynamics of the acoustic modes will be treated on the basis of a model of a metal hydride crystal slightly more general than $\mathrm{ZrH}_{2}$. It is as sumed that each proton has $n \geq 4$ nearest-neighbor metal atoms with which it interacts symmetrically, and that it interacts with no other atoms. The latter property is implied by the neglect of the width of the optical band, which is suggested, in the case of $\mathrm{ZrH}_{2}$, by the experimental result ${ }^{4}$ that the width of the optical transition peak is of the order of magnitude expected from Doppler broadening by acoustic motions only. It is further assumed that the metal-metal forces are less than, or at least no greater than, the proton-metal forces.

The Hamiltonian of the system is consequently taken to be

$$
\begin{aligned}
& H=T+V, \\
& T=\frac{1}{2} \sum_{i=1}^{N} \mathrm{k}_{i}^{2}+\frac{1}{2 M} \sum_{j=1}^{N^{\prime}} \mathbf{p}_{j}^{2}, \\
& V=\frac{a}{2} \sum_{i=1}^{N} \rho_{i}^{2}+U\left(\ldots \mathbf{q}_{j} \ldots\right),
\end{aligned}
$$

where

$$
\rho_{i}=\mathbf{r}_{i}-\frac{1}{n} \sum_{l=1}^{n} \mathbf{q}_{i l},
$$

and

$$
\begin{aligned}
T & =\text { kinetic energy, } \\
V & =\text { potential energy, } \\
\mathbf{r}_{i} & =\text { displacement of } i^{\text {th }} \text { proton from its equilibrium position, }
\end{aligned}
$$




\section{.}

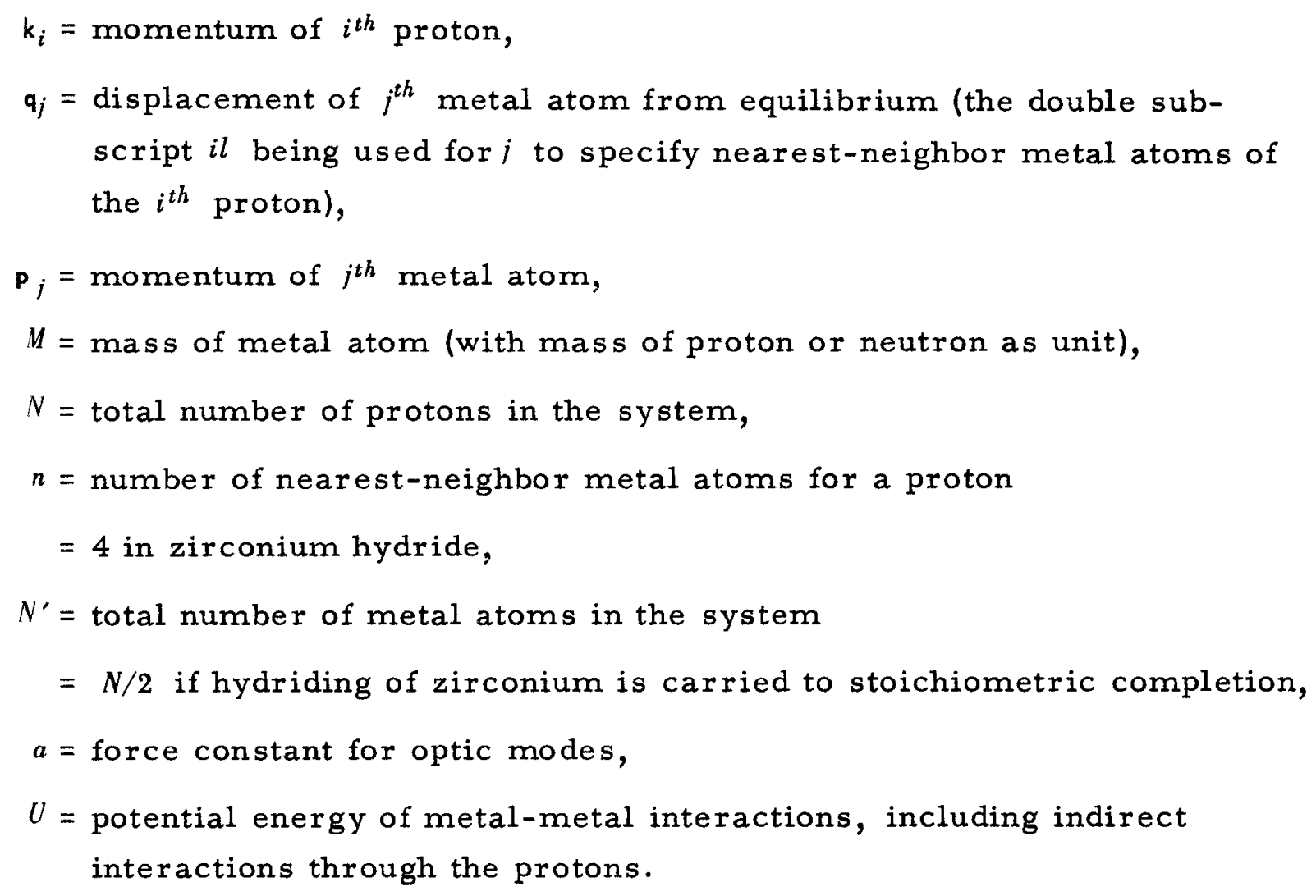
script $i l$ being used for $i$ to specify nearest-neighbor metal atoms of the $i^{\text {th }}$ proton),

The physical content of the se equations is in the absence from the potential energy, equation (3.3), of cross terms between the $\rho$ 's and $q$ 's. Since the coupling of each proton is only to its nearest neighbors, and is symmetric among them, such a cross term would have to be between $\rho_{i}$ and

$$
\sum_{l=1}^{n} \mathbf{q}_{i l}
$$

A displacement in which all the $q_{i l}$ were equal and non-vanishing would displace the equilibrium value of $\rho_{i}$ from zero if there were such a term. Since equation (4) defines $\rho_{i}$ as the displacement of the $i^{\text {th }}$ proton from the center of mass of its neighbor metal atoms, such a displacement of its equilibrium value must be prevented by suppressing the cross terms. 


\section{(AI}

If the momenta canonically conjugate to the $\rho_{i}$ are introduced, the kinetic energy is found not to separate. It is necessary to introduce new coordinates for the metal atoms by the transformation

$$
\phi_{j}=\mathbf{q}_{j}+\frac{1}{n M} \sum_{m=1}^{n(j)} \mathbf{r}_{j m},
$$

where $n(j)$ is the number of nearest-neighbor protons for the $j^{\text {th }}$ metal atom always eight for the stoichiometric composition $\mathrm{ZrH}_{2}-$ and the double subscript $j m$ indicates the nearest-neighbor protons of the $j^{\text {th }}$ metal atom.

Neglecting terms of order $q / M$ compared to $q$, and terms of order $r / M$ compared to $r$, the inverse transformation is

$$
\begin{aligned}
& \mathbf{r}_{i} \approx \rho_{i}+\frac{1}{n} \sum_{l=1}^{n} \phi_{i l}, \\
& \mathbf{q}_{j} \approx \phi_{j}-\frac{1}{n M} \sum_{m=1}^{n(j)} \rho_{j m} .
\end{aligned}
$$

To the same accuracy, the momenta conjugate to $\rho_{i}$ and $\phi_{i}$ are respectively

$$
\begin{aligned}
& \chi_{i} \approx k_{i}-\frac{1}{n M} \sum_{l=1}^{n} \rho_{i l}, \\
& \pi_{j} \approx p_{j}+\frac{1}{n} \sum_{m=1}^{n(j)} k_{j m},
\end{aligned}
$$




\section{AI}

and the inverse momentum transformation is

$$
\begin{aligned}
& \mathrm{k}_{i} \approx \boldsymbol{\chi}_{i}+\frac{1}{n M} \sum_{l=1}^{n} \pi_{i l}, \\
& \mathrm{p}_{i} \approx \pi_{i}-\frac{1}{n} \sum_{m=1}^{n(j)} \boldsymbol{\chi}_{j m^{.}}
\end{aligned}
$$

To the same accuracy as above, the kinetic energy now separates; in fact, the separation is exact if the inverse transformation is taken as exact, rather than the direct transformation:

$$
T \approx \frac{1}{2} \sum_{i=1}^{N} x_{i}^{2}+\frac{1}{2 M} \sum_{j=1}^{N^{\prime}} \pi_{j}^{2} .
$$

The Hamiltonian is not quite separated, however, since the coordinates $q_{j}$ in the metal-atom term $U\left(\ldots q_{j} \ldots\right)$ must be replaced by

$$
\phi_{j}-\frac{1}{n M} \sum_{m=1}^{n(j)} \rho_{j m}
$$

The order of magnitude of the cross terms is presumably $(a / M) p \cdot \phi$, the factor $a$ representing the assumption that the direct metal-metal forces of $U\left(\ldots q_{j} \ldots\right)$ do not dominate the indirect forces introduced into $U\left(\ldots q_{j} \ldots\right)$ by the transformation from the $r$ 's to the $\rho$ 's. In order to compare these cross terms with the terms containing $\rho$ 's or $\phi$ 's only, an estimate of the amplitude of the $\rho$ and $\phi$ oscillations is required. The virial theorem for a harmonic oscillator, to the effect that mean kinetic and potential energies are equal, permits such an estimate. In 


\section{(A)}

fact, it forces the mean values of $a \rho^{2}$ and $a q^{2}$ to be of the orders of magnitude of the mean energies of the optic modes and the acoustic modes respectively. These mean energies are sums of zero-point energies $h v / 2$ and thermal energies $k T$. The result is

$$
\frac{q}{\rho} \approx \sqrt{\frac{2 k T+(h \nu)_{a c}}{2 k T+(h \nu)_{o p}}}=\sqrt{\frac{2 k T}{(h \nu)_{o p}}},
$$

the last approximation being based on the frequency estimates for $\mathrm{ZrH}_{2}$ given in equations (1) and (2).

Clearly $\rho$ and $q$ are not of very different orders of magnitude, and the small factor $1 / M$ ensures that the cross terms make a minor contribution to the potential energy. It is consequently satisfactory to write

$$
V=\frac{a}{2} \sum_{i=1}^{N} \rho_{i}^{2}+U\left(\ldots \phi_{i} \ldots\right)
$$

The Hamiltonian now takes approximately a form in which the acoustic and optic modes are in separate terms; moreover, each proton is separated from all others:

$$
\begin{aligned}
H & \approx \sum_{i=1}^{N} H_{i}+H_{a c}, \\
H_{i} & =\frac{1}{2} X_{i}^{2}+\frac{a}{2} \rho_{i}^{2}, \\
H_{a c} & =\frac{1}{2 M} \sum_{j=1}^{N^{\prime}} \pi_{j}^{2}+U\left(\ldots \phi_{j} \ldots\right) .
\end{aligned}
$$




\section{M}

As a consequence of this separation of the Hamiltonian, it is known that the states of the system can be represented as products of states, each factor-state corresponding to a term of the Hamiltonian. The effect of including the acoustic modes in the analysis has been to add the term $H_{a c}$ to $H$, and thus to multiply the product of harmonic-oscillator states representing the state of the optic modes by a factor representing the state of the acoustic modes. 


\section{CALCULATION OF CROSS SECTIONS}

The "energy-loss" cross section for scattering a neutron of energy $E_{0}$ into the energy range $d E$ about $E_{f}$, with energy-loss

$$
\Delta E=E_{\mathrm{o}}-E_{f},
$$

is obtained by integrating over momentum transfers $\Delta k$ the "elementary" cross section for scattering with energy-loss $\Delta E$ and momentum transfer $\Delta k$. Integration over $\Delta k$ is equivalent to integration over neutron deflection angles, because of the relation

$$
\Delta k^{2}=2 E_{0}+2 E_{f}-4 \sqrt{E_{0} E_{f}} \mu,
$$

where $\mu$ is the cosine of the deflection angle.

The elementary cross section is proportional to the square of the matrix element of the operator $\exp \left(-i \Delta k \cdot r_{i}\right), \quad$ where $r_{i}$ is the position coordinate of the struck proton. Scattering by zirconium atoms has been neglected because the proton cross section is larger and protons are more numerous than zirconium atoms. Because the proton cross section is almost all incoherent, interference effects are ignored; thus, the macroscopic cross section is merely the product of the proton density by the microscopic cross section for a single proton, and the absence of a sum over $i$ in the above operator is justified.

In terms of the coordinates introduced in section III above, the operator may be written in the form

$$
\exp \left(-i \Delta k \cdot \rho_{i}\right) \exp \left(-\frac{i}{n} \Delta k \cdot \sum_{l=1}^{n} \phi_{i l}\right)
$$

This factoring of the operator, together with the corresponding factoring of the states of the system, results in a factoring of the matrix element and of its 


\section{$M$}

square. The elementary cross section consequently differs from that obtained by neglecting the acoustic modes by a factor

$$
\left|\left[\psi_{\beta}(a c), \exp \left(-\frac{\imath}{n} \Delta \mathrm{k} \cdot \sum_{l=1}^{n} \phi_{l l}\right) \psi_{a}(a c)\right]\right|^{2},
$$

where $\psi_{a}(a c)$ and $\psi_{\beta}(a c)$ denote the initial and final states of the acoustic modes.

In physical terms, these formulae mean that the cross section for a process, in which a neutron changes its momentum by $\Delta k$ and the proton state changes in some specified manner, must be multiplied by the probability that a transition between states of the acoustic modes is induced by the simultaneous transfer of momentum $\Delta k / n$ to each of the $n$ nearest-neighbor metal atoms of the struck proton.

It is best to partition the energy transfer $\Delta E$ into optical and acoustical parts, in the form

$$
\Delta E=\Delta E_{o p}+\Delta E_{a c}
$$

The cross section for the change of proton state then depends on $\Delta E_{o p}$, and the probability of the acoustic transition on $\Delta E_{a c^{*}}$. Since $\Delta E_{o p}$ can take on only values which are integer multiples of $h \nu_{o p}$, while $\Delta E_{a c}$ has a range, of the order of $k T$, that is much less than $h \nu_{o p}$, the value of $\Delta E$ determines the values of $\Delta E_{o p}$ and $\Delta E_{a c}$ all but uniquely. This uniqueness is really essential to the factoring of the cross section; if more than one partition of $\Delta E$ between $\Delta E_{o p}$ and $\Delta E_{a c}$ were to occur with significant probability, it would be necessary to sum the products corresponding to these partitions.

On the other hand, the states $\psi_{\alpha}(a c)$ and $\psi_{\beta}(a c)$ are not uniquely determined by the value of $\Delta E_{a c}$; consequently, it is necessary to average the square of the acoustic matrix element over the initial states of the acoustic modes, and sum 
over the final states, subject to the condition that the energy transfer have the correct value $\Delta E_{a c}$. The initial states $\psi_{a}(a c)$ are weighted in the averaging by their Boltzmann factors $\exp \left(-E_{\alpha} / k T\right)$.

The probability that the energy transfer is $\Delta E_{a c}$ is then given by

$$
\begin{aligned}
P\left(\Delta E_{a c}, \Delta \mathrm{k}\right)= & \sum_{\beta} \delta\left(E_{a}-E_{\beta}+\Delta E_{a c}\right) \\
& \frac{\sum_{a} \exp \left(-\frac{E_{a}}{k T}\right)\left|\left[\psi_{\beta}(a c), \exp \left(-\frac{i}{n} \Delta \mathrm{k} \cdot \sum_{l=1}^{n} \phi_{i l}\right) \psi_{a}(a c)\right]\right|^{2}}{\sum_{a} \exp \left(-\frac{E_{a}}{k T}\right)} .
\end{aligned}
$$

As noted by several authors ${ }^{6,7}$ in similar problems, the sums over states in the numerator are much easier if $P\left(\Delta E_{a c}, \Delta k\right)$ is Fourier transformed with respect to the variable $\Delta E_{a c}$. The sums thus obtained may be evaluated readily by virtue of the completeness of the set of states, the resulting expression for the Fourier transform of $P$ being

$$
\begin{aligned}
Q(t, \Delta k)= & \frac{\operatorname{Tr}\left[\exp \left(-\frac{H_{a c}}{k T}+i H_{a c} t\right) \cdot \exp \left(\frac{i}{n} \Delta \mathrm{k} \cdot \sum_{l=1}^{n} \phi_{i l}\right) \cdot \exp \left(-i H_{a c} t\right) \cdot \exp \left(-\frac{i}{n} \Delta \mathrm{k} \cdot \sum_{l=1}^{n} \phi_{i l}\right)\right]}{T_{r}\left[\exp \left(-\frac{H_{a c}}{k T}\right)\right]} \\
& =\frac{\operatorname{Tr}_{r}\left\{\exp \left(-\frac{H_{a c}}{k T}+i H_{a c} t\right) \cdot \exp \left[-i t H_{a c}\left(\ldots \pi_{i l}+\frac{\Delta \mathrm{k}}{n} \ldots\right)\right]\right\}}{T_{r}\left[\exp \left(-\frac{H_{a c}}{k T}\right)\right]} . .(10)
\end{aligned}
$$




\section{Ar}

The notation $H_{a c}\left(\ldots \pi_{i l}+\Delta \mathrm{k} / n \ldots\right)$ indicates that the $n$ arguments $\pi_{i l}$ of $l_{a c}$ are all incremented by $\Delta k / n$, while the other arguments are left unchanged.

The evaluation of $Q(t, \Delta k)$ is greatly simplified by the use of the classical approximation. This approximation is justified if the thermal energy $k T$ is considerably larger than the quantum energy $(h \nu)_{a c}$. An earlier estimate has suggested, however, that $k T$ is abrut twice $(h v)_{a c}$. This estimate applies, of course, to the higher-frequency modes; there are certainly modes of lower frequency for which the classical approximation is valid. Unfortunately, most of the modes are no doubt of relatively high frequency. It should be recalled, however, that for many purposes the classical approximation is not bad even for small quantum numbers; its principle deficiency in the present case is probably that it allows a continuous rather than a discrete range of energies for a single normal mode. In an infinite crystal, however, the normal modes themselves constitute a continuum, and the range of energies available to the system as a whole is therefore actually continuous. The fact that a small change of the energy considered requires consideration of a different, neighboring normal mode may be expected to make relatively little difference. It seems probable, then, that the classical approximation will be fairly reliable in the present case. It may be noted, moreover, that the conclusions to be drawn do not require an estimate of great accuracy.

In the classical approximation, the potential and kinetic energy terms in the Hamiltonian commute. Consequently, the addition theorem for the exponential function is valid for the exponential of $H_{a c}$. This permits a great many concellations in the formula for $Q(t, \Delta \mathbf{k})$; in particular, the potential energy $U\left(\ldots \phi_{j} \ldots\right)$ drops out, and only the kinetic energy term of $H_{a c}$ need be retained. This means that the effect of the acoustic modes on the scattering in the classical approximation is as if the metal atoms were not mutually coupled.

These effectively free metal atoms may be divided into the $n$ nearest neighbors of the struck proton, each of which receives the momentum increment $\Delta k / n$, and all other metal atoms. The latter, like the potential energy, cancel out of the expression for $(t, \Delta k)$. As for the $n$ that remain, the index il which identifies them actually identifies the dummy variables $\pi_{l} l^{;}$it doesn't really matter, therefore, which $n$ metal atoms are accelerated. This is a consequence of the neglect of interference effects. 


\section{$1 /$}

Thus, $Q(t, \Delta k)$ is the $n^{t h}$ power of the expression for a single free metal atom:

$$
Q(t, \Delta k)=\left[\frac{\operatorname{Tr}\left\{\exp \left[\left(i t-\frac{1}{k T}\right) \frac{\pi^{2}}{2 M}\right] \cdot \exp \left[-\frac{i t}{2 M}\left(\pi+\frac{\Delta k}{n}\right)^{2}\right]\right\}}{\operatorname{Tr}\left[\exp \left(-\frac{\pi^{2}}{2 M k T}\right)\right]}\right]^{n}
$$

In this expression, the trace is to be interpreted classically as an integration over momentum space. By completing the square in the exponent of the trace in the numerator, the traces in numerator and denominator can be made to cancel each other, leaving the result

$$
\begin{aligned}
Q(t, \Delta k) & =\left\{\exp \left[-\left(i t+k T t^{2}\right) \frac{(\Delta k / n)^{2}}{2 M}\right]\right\}^{n} \\
& =\exp \left[-\left(i t+k T t^{2}\right) \frac{\Lambda_{k}^{2}}{2 n M}\right] .
\end{aligned}
$$

This is the known ${ }^{8}$ Fourier transform of the square of the matrix element for scattering by a free particle of mass $n M$ with momentum transfer $\Delta k$. The $n$ metal atoms of mass $M$ each, which receive the momentum $\Delta k$ when the proton recoils in the collision, act like a single free particle of mass nM. The actual process does indeed involve the transfer of momentum $\Delta k$ to a system of $n$ atoms whose total mass is $n M$, but this system is fairly complex. It appears from the formalism that the complexity is not significant because of the complete absence of correlations between the initial states of these $n$ atoms, the coupling through the proton of which they are nearest neighbors, which could produce such correlations, having dropped out with the potential energy as a consequence of the classical approximation. 


\section{MODIFIED WILKINS EQUATION FOR ACOUSTIC MODES}

Before completing the evaluation of the energy-loss cross section by integrating the elementary cross sections over the neutron deflection angles, it is still necessary to invert the Fourier transform $Q(t . \Delta k)$ to obtain the acoustic probability factor $P\left(\Delta E_{a c}, \Delta k\right)$. The integration is somewhat intricate, however, and the results are not very perspicuous. These difficulties may be reduced by expanding $Q(t, \Delta k)$ in powers of $1 / M$ before carrying out the Fourier inversion, getting

$$
Q(t, \Delta k) \approx 1-\left(i t+k T t^{2}\right) \frac{\Delta k^{2}}{2 n M}
$$

As this procedure has been used ${ }^{8}$ to derive the Wilkins equation for the neutron spectrum in a heavy-gas moderator, it will yield something akin to the Wilkins equation in the present case also.

The Fourier inversion of the polynomial in $t$ by which $Q$ has been approximated results in a representation of $P$ by means of the $\delta$-function and its derivatives:

$$
P\left(\Delta E_{a c}, \Delta k\right) \approx\left\{1+\frac{\Delta k^{2}}{2 n M}\left[-\frac{d}{d\left(\Delta E_{a c}\right)}+k T \frac{d^{2}}{d\left(\Delta E_{a c}\right)^{2}}\right]\right\} \delta\left(\Delta E_{a c}\right)
$$

Since the first term is independent of $\Delta k$, the integration over $\Delta k$ proceeds as if there were no acoustic modes, and the result, including the factor $\delta\left(\Delta E_{a c}\right)$, is just what it would have been without these modes. The term containing $\Delta k^{2}$ is less trivial, however.

The case of most interest is that in which the neutron initial energy is too low to excite the optical modes. The cross section for elastic scattering by the isotropic oscillator is then 


$$
\begin{aligned}
& \text { (A) } \\
& \sigma_{S H O}=2 \sigma_{0} \sqrt{\frac{E_{f}}{E_{0}}} \int_{-1}^{1} d \mu \exp \left(-\frac{\Delta k^{2}}{2 \omega}\right),
\end{aligned}
$$

with $\Delta k$ and $\mu$ related according to equation (8), $\omega$ representing the quantum energy of the oscillator, and $\sigma_{0}$ the neutron cross section of a free proton.

The effect of the acoustic modes is to introduce the factor $P\left(\Delta E_{a c}, \Delta k\right)$ into the integrand of the last expression, yielding for the energy-loss cross section an expression of the form

$$
\frac{d \sigma}{d E_{f}}=2 \sigma_{0} \sqrt{\frac{E_{f}}{E_{0}}} \int_{-1}^{1} d \mu \exp \left(-\frac{\Delta k^{2}}{2 \omega}\right)\left\{1+\frac{\Delta k^{2}}{2 n M}\left[-\frac{d}{d(\Delta E)}+k T \frac{d^{2}}{d(\Delta E)^{2}}\right]\right\} \delta(\Delta E),
$$

in which $\Delta E_{a c}$ has been replaced by $\Delta E$ because $\Delta E_{o p}$ vanishes for the transition being considered.

The differentiation of the $\delta$-function will ultimately be interpreted by integrating by parts; it will then appear that not merely the values of all functions at $\Delta E=0$ are significant, as suggested by the factor $\delta(\Delta E)$, but also the values of their first derivatives with respect to $\Delta E$. An equivalent statement is that the first two terms of a power series in $\Delta E$ are needed.

The elimination of $\Delta k$ may be performed by writing equation ( 8 ) in the form

$$
\begin{aligned}
\Delta k^{2} & =2\left[E_{0}+E_{f}-2 \sqrt{E_{0} E_{f}}\right]+4 \sqrt{E_{0} E_{f}}(1-\mu) \\
& =2\left(\sqrt{E_{0}}-\sqrt{E_{f}}\right)^{2}+4 \sqrt{E_{0} E_{f}}(1-\mu)
\end{aligned}
$$


and noting that

$$
\Delta E=E_{0}-E_{f}=\left(\sqrt{E_{0}}-\sqrt{E_{f}}\right)\left(\sqrt{E_{0}}+\sqrt{E_{f}}\right) \approx 2 \sqrt{E_{0}}\left(\sqrt{E_{0}}-\sqrt{E_{f}}\right),
$$

whence there follows

$$
\Delta k^{2} \approx 4 \sqrt{E_{0} E_{f}}(1-\mu)
$$

This approximation is correct to order $\Delta E$, and thus, according to the preceding paragraph, will lead to exact results for the cross section. The exponential in equation (14) may be written, to the same accuracy, as

$$
\exp \left(-\frac{\Delta \varepsilon^{2}}{2 \omega}\right) \approx \exp \left[-2 \frac{\sqrt{E_{0} E_{f}}}{\omega}(1-\mu)\right]
$$

With these substitutions, the cross section may be presented in the form

$$
\frac{d \sigma}{d E_{f}}=\left[\sigma_{s}\left(\frac{E_{0}}{\omega}\right)+\frac{1}{n M} 2 E_{f_{t}}\left(\frac{\sqrt{E_{0} E_{f}}}{\omega}\right)\left(-\frac{d}{d \Delta E}+k T \frac{d^{2}}{d \Delta E^{2}}\right)\right] \delta(\Delta E),
$$

where

$$
\begin{aligned}
\sigma_{s}\left(\frac{E_{0}}{\omega}\right) & =2 \sigma_{0} \int_{-1}^{1} d \mu \exp \left[-2 \frac{E_{0}}{\omega}(1-\mu)\right] \\
& =\sigma_{0} \frac{\omega}{E_{0}}\left[1-\exp \left(-\frac{4 E_{0}}{\omega}\right)\right],
\end{aligned}
$$




$$
\begin{aligned}
\sigma_{t}\left(E_{0} / \omega\right)= & 2 \sigma_{0} \int_{-1}^{1} d \mu(1-\mu) \exp \left[-2 \frac{E_{0}}{\omega}(1-\mu)\right] \\
= & \frac{\sigma_{0}}{2}\left(\frac{\omega}{E_{0}}\right)^{2}\left[1-\left(1+\frac{4 E_{0}}{\omega}\right) \exp \left(-\frac{4 E_{0}}{\omega}\right)\right] .
\end{aligned}
$$

The notation indicates that $\sigma_{s}\left(E_{0} / \omega\right)$ and $\sigma_{t}\left(E_{0} / \omega\right)$ are the scattering and transport cross sections, respectively, of an isotropic-oscillator proton of frequency $\omega$.

The neutron flux is determined by an integral equation representing the neutron balance, having $d o / d E_{f}$ for its kernel. Hurwitz, Nelkin, and Habetler ${ }^{8}$ have shown how this equation reduces to a differential equation when, as in equation (15), the kernel is expressed in terms of the $\delta$-function and its derivatives; the coefficients of the equation are the first and second moments of the energy loss. Following the method of these authors, the neutron flux $\phi(E)$ is replaced by the ratio $\psi(E)$ between $\phi(E)$ and the Maxwellian corresponding to the moderator temperature:

$$
\psi(E)=\phi(E) E^{-1} \exp \left(\frac{E}{k T}\right)
$$

The equation satisfied by $\psi(E)$ is then found to be

$$
k T E \psi^{\prime \prime}-\left[E-2 k T \frac{4 \sigma_{0} \exp (-4 E / \omega)}{\sigma_{t}(E / \omega)}\right] \psi^{\prime}-\frac{\sigma_{a}(E)}{\xi \sigma_{t}(E / \omega)} \psi+S(E)=0
$$

where $\xi=2(n H)^{-1}$ is the mean logarithmic energy loss for a moderator of mass $n M, \sigma_{a}(E)$ is the absorption cross section of the medium per proton, and $S(E)$ is a term, representing neutrons arriving in or departing from the energy range $d E$ at $E$ by means of inelastic optical transitions, whose detailed structure will not be required. 


\section{(AI)}

The derivation of this result ${ }^{8}$ exploits the "detailed balancing" property of the energy-loss cross section. This property, although very general, is not quite obvious in the present case because it applies to the entire cross section, whereas it is here applied to a partial cross section; namely, the cross section for a process in which the proton oscillations are not excited. In principle, the same energy transfer could be achieved by a process in which the proton is excited, the energy for this purpose being drawn from the acoustic modes. Since such an event is unlikely at reasonable temperatures, detailed balancing is expected to apply with sufficient accuracy to the partial process actually considered.

It is well known, and may be confirmed by examination of equation (15.2), that the transport cross section $\sigma_{t}(E / \omega)$ approaches $4 \sigma_{0}$ as $E / \omega$ approaches zero. This is the limit of infinite frequency $\omega$, representing completely rigid binding of the proton to its neighbor metal atoms. In this limit, equation (17) becomes

$$
k T E \psi^{\prime \prime} . .(E-2 k T) \psi^{\prime}-\frac{\sigma_{a}(E)}{\xi \cdot 4 \sigma_{o}} \dot{\psi}=0
$$

which is the Wilkins equation for a monatomic gas moderator of cross section $4 \sigma_{0}$ and mass $n M$; the term $S(E)$ has been dropped, since the optical modes cannot be excited in the limit of infinite frequency.

The factor

$$
\frac{4 \sigma_{0} \exp (-4 E / \omega)}{\sigma_{t}(E / \omega)}
$$

has been calculated for the case of zirconium hydride at room temperature, for which $n=4, M=91.22$, and $\omega=5.2 k 7$. From the value unity at $E=U$, it decreases to 0.74 at $E=k T$, and further to 0.16 at $E=\omega$. The correction term proportional to $2 k T$ in the coefficient of $\psi^{\prime}$ is thus seen to be less significant in zirconium hydride than in a heavy monatomic gas. 


\section{(AI)}

The relative importance of absorption and moderation is measured by the coefficient of $\psi$ in equation (17). It is of interest that the effectiveness of the moderator is governed by the transport cross section $\sigma_{t}(E / \omega)$ rather than by the scattering cross section $\sigma_{s}(E / \omega)$. The quantity $\xi \sigma_{t} / \sigma_{a}$, which is the reciprocal of the coefficient of $\psi$, is a rough estimate of the mean logarithmic energy loss to absorption for a neutron of energy $E$ which suffers no further optically inelastic collisions.

This quantity has been calculated for zirconium hydride at room temperature with a $1 / v$ absorber added to give a total of $2.5 b$ of absorption per proton at $2200 \mathrm{~m} \mathrm{sec}^{-1}$; this corresponds to enough $\mathrm{U}^{235}$ to give 320 times as many protons as uranium atoms. The values of $\xi \sigma_{t} / \sigma_{a}$ are 0.11 if $E=k T$ and 0.046 if $E=\omega$. These values mean that a neutron may lose 5 or 10 percent of its energy to the acoustic modes before being absorbed. Such losses seem small enough to justify neglect of acoustic modes in estimating the neutron spectrum in a system of the composition given above.

The weakest point in the argument leading to this conclusion is the use of the classical approximation in deriving equation (11) from equation (10). The uncertainties arising from this approximation are increased by the somewhat speculative frequency estimates used to help justify it. It is unlikely, however, that the above estimates of energy loss to absorption would be increased more than 50 percent by improving this aspect of the argument, and such an increase would not seriously upset the conclusion that acoustic effects can be ignored. 


\section{REFERENCES}

1. E. U. Vaughan and E. R. Cohen, NAA-SR-3376, January 15, 1959.

2. E. R. Cohen and E. U. Vaughan, NAA-SR-3377, January 15, 1959.

3. I. Pelah et al., Phys. Rev. 108, 1091 (1957).

4. A. Andresen et al., Phys. Rev. 108, 1092 (1957).

5. R. E. Rundle, C. G. Shull, and E. O. Wollan, AECU-1275 (April 1951); Acta Cryst. 5, 22 (1952).

6. G. C. Wick, Phys. Rev. 94, 1228 (1954).

7. A. C. Zemach and R. J. Glauber, Phys. Rev. 101, 118 and 129 (1956).

8. H. Hurwitz, Jr., M. S. Nelkin, and G. J. Habetler, Nuclear Sci. and Eng. 1, 280 (1956). 